Pacific Journal of Mathematics

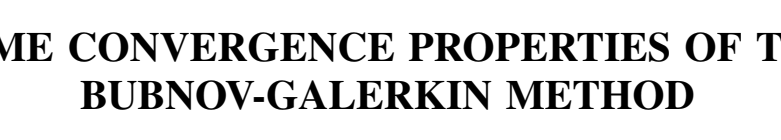




\title{
SOME CONVERGENCE PROPERTIES OF THE BUBNOV-GALERKIN METHOD
}

\author{
S. R. SINGH
}

We generalize the Bubnov-Galerkin method to approximate the resolvent of the $m$-sectorial operator associated with a densely defined, closed, sectorial form in a Hilbert space. Some special cases of interest are also discussed.

1. Introduction. The Bubnov-Galerkin method [3] was originally devised to approximate the solutions of the equations of the form

$$
(z-A) f=g
$$

where $A$ is an operator in a Hilbert space, $\mathscr{H}, g$ is a vector in $\mathscr{H}$ and $z$ is a complex number. The method proceeds with solving the following set of equations:

$$
\sum_{j=1}^{n} \alpha_{j}\left(\phi_{i} \mid(z-A) \phi_{j}\right)=\left(\phi_{i} \mid g\right) \quad i=1, \cdots, n
$$

where (.|.) denotes the scalar product in $\mathscr{H}$ and $\left\{\phi_{i}\right\} \subset \mathscr{D}(A)$ is some linearly independent (l.i.) set in $\mathscr{H} \cdot \mathscr{D}(\cdot)$ denotes the domain. The questions of interest are the existence and the convergence of the solutions of equation (2). Until recently, the only cases that received a detailed treatment have been when $A$ is compact, bounded or essentially self-adjoint $[3,6]$. However, recently the following result was proven by Masson and Thewarapperuma [2]:

R.1. Let $A$ be symmetric, bounded below by $b, z$ be at a nonzero distance from $[b, \infty)$ and $\left\{\phi_{i}\right\}$ be the orthonormal set formed from $\left\{A^{i} h\right\}$ where $h$ is in $\mathscr{D}\left(A^{i}\right)$ for each $i$. Then $\lim _{n \rightarrow \infty} \| \sum_{j=1}^{n} \alpha_{j} \phi_{j}-$ $\left(z-A_{F}\right)^{-1} g \|=0$, where $\|$.$\| denotes the norm in \mathscr{H}$ and $A_{F}$ is the Friedrichs extension of $A$.

Consider the following set of equations:

$$
\sum_{j=1}^{n} \alpha_{j}\left[z\left(\phi_{i} \mid \phi_{j}\right)-t\left(\phi_{i}, \dot{\phi}_{j}\right)\right]=\left(\phi_{i} \mid g\right) \quad i=1, \cdots, n
$$

where $t$ is a densely defined, closable, sectorial, sesquilinear form in $\mathscr{H}$. The sector of $t$ will be denoted by $S$ and since it causes no loss of generality, the vertex will be taken to be one. In the present note we determine the limit of $f_{n}=\sum_{j=1}^{n} \alpha_{j} \phi_{j}$ as $n$ becomes large. 
R.1. and some other generalizations of it, will follow from our main result (Theorem 1).

2. Results. Define a new scalar product $(. \mid .)_{t}$ on $\mathscr{D}(t)$ by $(u \mid v)_{t}=\operatorname{Re} \cdot t(u, v),[1$, pp. 309-10] and complete $\mathscr{D}(t)$ in the new metric to a Hilbert space $\mathscr{K}_{t}$. Let the closure of $t$ be $\bar{t}$. We have that $\mathscr{D}(t) \subset \mathscr{D}(\bar{t})=\mathscr{H}_{t} \subset \mathscr{H}$. The norm in $\mathscr{H}_{t}$ will be denoted by $\|\cdot\|_{t}$. Also $\mathscr{B}(X, Y)$ will denote the space of bounded operators with $\mathscr{D}(\cdot) \subset X$ and range $\mathscr{R}(\cdot) \subset Y$, and $\mathscr{B}(X)=\mathscr{B}(X, X)$.

LEMma 1. Let $t$ be as in equation (3), $\left\{\phi_{i}\right\} \subset \mathscr{D}(t)$ and $g \in \mathscr{H}$. Equation (3) is equivalent to

$$
\sum_{j=1}^{n} \alpha_{j}\left(\phi_{i} \mid[1-T(z)] \dot{\phi}_{j}\right)_{t}=-\left(\phi_{i} \mid B g\right)_{t} \quad i=1, \cdots, n ;
$$

where $B \in \mathscr{B}\left(\mathscr{H}, \mathscr{H}_{t}\right), \quad T(z)=\left(z B_{t}-C\right) \in \mathscr{B}\left(\mathscr{H}_{t}\right)$ and $B_{t}$ is the restriction of $B$ to $\mathscr{D}(t)$.

Proof. Since $t_{1}=(t-$ Re. $t)$ is a bounded form on $\mathscr{H}_{t}[1$, p. 314], there is a $C \in \mathscr{B}\left(\mathscr{H}_{t}\right)$ such that

$$
t_{1}(u, v)=(u \mid C v)_{t} ; u, v \in \mathscr{D}(t) .
$$

Also from Ref. [4] pp. 332-3, it follows that there is a unique $B \in$ $\mathscr{B}\left(\mathscr{H}, \mathscr{H}_{t}\right)$ such that $\mathscr{D}(B)=\mathscr{H}$ and for $u \in \mathscr{H}_{t}, w \in \mathscr{H}$,

$$
(u \mid \omega)=(u \mid B \omega)_{t} .
$$

In particular, in equation (3), $\left(\phi_{i} \mid g\right)=\left(\dot{\phi}_{i} \mid B g\right)_{t}$ and $\left(\dot{\phi}_{i} \mid \dot{\phi}_{j}\right)=$ $\left(\phi_{i} \mid B \phi_{j}\right)_{t}=\left(\phi_{i} \mid B_{t} \dot{\phi}_{j}\right)_{t}$.

The assertion now follows from direct substitution.

LEMMa 2. In the notation of Lemma 1 , we have that $B_{t}$, $C$ are closable, $B$ is closed and invertible and $B^{-1}(1+\bar{C})=A_{t}$ where $A_{t}$ is the unique m-sectorial operator associated with $\bar{t}$.

Proof. Since $B_{t}$ and $C$ are bounded and densely defined, they are closable. Since $B$ is bounded and $\mathscr{D}(B)=\mathscr{H}$, it is closed. Invertibility of $B$ has been proven in Reference [4] p. 333.

Now, $\mathscr{D}\left(\left[B^{-1}(1+\bar{C})\right]\right) \subset \mathscr{H}_{t}=\mathscr{D}(\bar{t})$ and for $u, v \in \mathscr{D}(t)$,

$$
\begin{aligned}
\left(u \mid B^{-1}(1+\bar{C}) v\right) & =\left(u \mid B^{-1}(1+C) v\right) \\
& =(u \mid(1+C) v)_{t} \\
& =t(u, v)
\end{aligned}
$$

From the closability of $t$, this result extends for $u, v \in \mathscr{H}_{t}$. The 
result now follows from Theorem 2.1, Chapter 6, Reference [1].

THEOREM 1. In addition to the assumptions of Lemma 1 and 2 , let $\left\{\phi_{i}\right\}$ be 1.i. and complete in $\mathscr{H}_{t}$, and $z$ be at a nonzero distance from $S . f_{n}=\sum_{j=1}^{n} \alpha_{j} \phi_{j}$ of equation (3) is then defined for each $n$ and $\lim _{n \rightarrow \infty}\left\|f_{n}-\left(z-A_{t}\right)^{-1} g\right\|=0$.

Proof. From Lemma 1, equation (3) is equivalent to equation (4). Also without loss of generality, we may assume $\left\{\phi_{i}\right\}$ to be an orthonormal basis in $\mathscr{H}_{t}$. It is straightforward to check that (4) is equivalent to

$$
\left(1-T_{n}(z)\right) f_{n}=-P_{n} B g
$$

where $T_{n}(z)=P_{n} T(z) P_{n}$, and $P_{n}$ is the ortho-projection on the $n$ dimentional subspace of $\mathscr{H}_{t}$ determined by $\left\{\phi_{i}\right\}, i=1$ to $n$. It follows, for $h \in \mathscr{H}_{t}$, that

$$
\lim _{n \rightarrow \infty}\left\|\left(T_{n}(z)-\bar{T}(z)\right) h\right\|_{t}=0 .
$$

Also, since $z$ is at a nonzero distance from $S$, dist. $(1, W(\bar{T}(z)))=$ $d^{\prime}>0$, where $W(\cdot)$ denotes the numerical range. Further, since the spectrum of $T_{n}, \sigma\left(T_{n}\right) \subset(W(\bar{T}(z)) \cup\{0\})$, for each $n,\left(1-T_{n}(z)\right)^{-1} \in$ $\mathscr{B}\left(\mathscr{C}_{t}\right)$ with $\left\|\left(1-T_{n}(z)\right)^{-1}\right\|_{t} \leqq 1 / d \quad$ where $d=\min .\left(1, d^{\prime}\right)$. Also $(1-\bar{T}(z))^{-1} \in \mathscr{B}\left(\mathscr{H}_{t}\right)$.

Hence for $h \in \mathscr{H}_{t}$

$$
\begin{aligned}
\|[(1- & \left.\left.T_{n}(z)\right)^{-1}-(1-\bar{T}(z))^{-1}\right] h \|_{t} \\
= & \left\|\left(1-T_{n}(z)\right)^{-1}\left(T_{n}(z)-\bar{T}(z)\right)(1-\bar{T}(z))^{-1} h\right\|_{t} \\
& \leqq \\
& \underset{n \rightarrow \infty}{\longrightarrow} 0 .
\end{aligned}
$$

Further, for $g \in \mathscr{H}$,

$$
\lim _{n \rightarrow \infty}\left\|\left(P_{n} B-B\right) g\right\|_{t}=0
$$

and hence

$$
\lim _{n \rightarrow \infty}\left\|f_{n}-f\right\|_{t}=0
$$

where

$$
\begin{aligned}
f=-(1-\bar{T}(z))^{-1} B g & =-\left(1-z \bar{B}_{t}+\bar{C}\right)^{-1} B g \\
& =\left(z-B^{-1}(1+\bar{C})\right)^{-1} g \\
& =\left(z-A_{t}\right)^{-1} g \quad(\text { Lemma 2) }
\end{aligned}
$$


Assertion of the theorem follows by observing that $\|\cdot\|_{t} \geqq\|\cdot\|$. For a symmetric $t, s=[b, \infty)$ with some $b>-\infty, \bar{C}=0$ and $A_{t}=B^{-1}$ is self-adjoint.

In the following, $f_{n}$ will stand for $\sum_{j=1}^{n} \alpha_{j} \phi_{j}$ as defined by equation (2).

COROLlaRy 1. Let $A$ be densely defined sectorial operator and $z$ be at a nonzero distance from its sector, $\left\{\phi_{i}\right\}$ be a l.i. basis in $\mathscr{D}(A)$. We have that $\lim _{n \rightarrow \infty}\left\|f_{n}-\left(z-A_{F}\right)^{-1} g\right\|=0$.

Proof. Define $t$ of Theorem 1 by $t(u, v)=(u \mid A v), u, v \in \mathscr{D}(A)$. $t$ is closable from Theorem 1.27, Chapter 6 of [1]. Since $\left\{\phi_{i}\right\}$ is a 1.i. basis in $\mathscr{D}(A)$ and $\mathscr{D}(A)$ is dense in $\mathscr{D}(\bar{t})=\mathscr{H}_{t}$, it is a l.i. basis in $\mathscr{H}_{t}$. The result now follows from the fact that $A_{t}$ of Theorem 1 now becomes $A_{F}$ [1, pp. 325-6].

COROLlaRy 2. Let $A$ be symmetric, bounded below by $b, z$ be at a nonzero distance from $[b, \infty)$ and $\left\{\phi_{i}\right\}$ be a 1.i. basis in $\mathscr{D}(A)$. Then $\lim _{n \rightarrow \infty}\left\|f_{n}-\left(z-A_{F}\right)^{-1} g\right\|=0$.

Proof. The result follows from Corollary 1, by noticing that the sector of $A$ is $[b, \infty)$.

If the set $\left\{\phi_{i}\right\}$ is taken to be $\left\{A^{i} h\right\}$ for some $h \in \mathscr{D}\left(A^{i}\right)$ for $i=$ $0,1,2, \cdots$; the Bubnov-Galerkin method is called the method of moments [7]. Since $\left\{A^{i} h\right\}$ satisfies the conditions of Corollaries 1 and 2 , the convergence of the method of moments also is established by these results. The result R.1 [2] thus is a special case of Corollary 2.

In Corollaries 1 and 2 we have considered the case of a densely defined $A$. In these results one can replace this condition by requiring that the form domain of $A$ be dense. However since the Friedrichs extension is defined only for a densely defined $A$, the limit operator $A_{t}$ may not be $A_{F}$. This situation is of a particular interest in Physics which we describe in brief.

Let $A$ be given, formally, by $A=A_{1}+A_{2}$, where $A_{1}$ and $A_{2}$ are symmetric but $\mathscr{D}(A)=\mathscr{D}\left(A_{1}\right) \cap \mathscr{D}\left(A_{2}\right)$ is not dense. However if the form domain of $A$ is dense, the self-adjoint operator $A_{t}$ associated with the form $t(u, v)=\left(u \mid\left(A_{1}+A_{2}\right) v\right)$ is a legitimate operator to describe a physical system [5]. This construction enables one to include a larger class of interactions in the treatment than the requirement that $A$ be densely defined [5]. It is obvious that the Bubnov-Galerkin method enables one to compute the resolvent of $A_{t}$ in this case also, which is of prime importance in Physics. 
Acknowledgement. The author is thankful to Professor J. Nuttall for helpful discussions and his hospitality.

\section{REFERENCES}

1. T. Kato, Perturbation Theory for Linear Operators, Springer Verlag, N.Y., 1966.

2. D. Masson and P. Thewarapperuma, On a connection between the method of moments and the Friedrichs extension, University of Toronto Preprint.

3. S. G. Mikhlin, Variational Methods in Mathematical Physics, Pergamon Press, N.Y., 1964, ch. IX.

4. F. Riesz and B. Sz. Nagy, Functional Analysis; Unger, N.Y., 1971.

5. B. Simon, Quantam Mechanics for Hamiltonians Defined as Quadratic forms, Princeton University Press, 1971, Ch. 2.

6. S. R. Singh and A. D. Stauffer, Nuovo Cimento 22B (1974) pp. 139-52.

7. Yu. V. Vorobyev, Method of Moments in Applied Mathematics, Gordon and Breach, N.Y., 1965.

Received February 10, 1976. Work supported in part by the National Research Council of Canada and the Centre for Interdisciplinary Studies in Chemical Physics, University of Western Ontario.

UNIVERSITY OF WESTERN ONTARIO 



\section{PACIFIC JOURNAL OF MATHEMATICS}

\section{EDITORS}

RICHARD ARENS (Managing Editor) University of California

Los Angeles, California 90024

R. A. Beaumont

University of Washington

Seattle, Washington 98105
J. DUGUNDJI

Department of Mathematics University of Southern Californı

Los Angeles, California 90007

D. Gilbarg and J. Milgram

Stanford University

Stanford, California 94305

\section{ASSOCIATE EDITORS}
E. F. BECKENBACH
B. H. NeumanN
F. WOLF
K. YosHIDA

\section{SUPPORTING INSTITUTIONS}

UNIVERSITY OF BRITISH COLUMBIA

CALIFORNIA INSTITUTE OF TECHNOLOGY

UNIVERSITY OF CALIFORNIA

MONTANA STATE UNIVERSITY

UNIVERSITY OF NEVADA

NEW MEXICO STATE UNIVERSITY

OREGON STATE UNIVERSITY

UNIVERSITY OF OREGON

OSAKA UNIVERSITY
UNIVERSITY OF SOUTHERN CALIFORNIA

STANFORD UNIVERSITY

UNIVERSITY OF TOKYO

UNIVERSITY OF UTAH

WASHINGTON STATE UNIVERSITY

UNIVERSITY OF WASHINGTON

* * * *

AMERICAN MATHEMATICAL SOCIETY

NAVAL WEAPONS CENTER 


\section{Pacific Journal of Mathematics \\ Vol. 65, No. $1 \quad$ September, 1976}

David Lee Armacost, Compactly cogenerated LCA groups ............. 1

Sun Man Chang, On continuous image averaging of probability measures ...... 13

J. Chidambaraswamy, Generalized Dedekind $\psi$-functions with respect to a

polynomial. II................................... 19

Freddy Delbaen, The Dunford-Pettis property for certain uniform algebras ..... 29

Robert Benjamin Feinberg, Faithful distributive modules over incidence

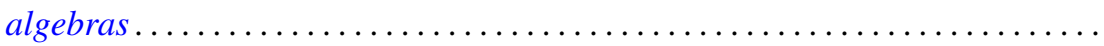

Paul Froeschl, Chained rings . . . . . . . . . . . . . . . . . . . .

John Brady Garnett and Anthony G. O'Farrell, Sobolev approximation by a sum

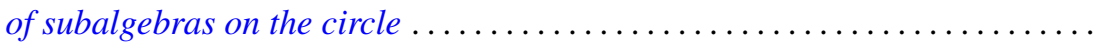

Hugh M. Hilden, José M. Montesinos and Thomas Lusk Thickstun, Closed

oriented 3-manifolds as 3-fold branched coverings of $S^{3}$ of special type.....

Atsushi Inoue, On a class of unbounded operator algebras ................

Peter Kleinschmidt, On facets with non-arbitrary shapes.

Narendrakumar Ramanlal Ladhawala, Absolute summability of Walsh-Fourier

series

Howard Wilson Lambert, Links which are unknottable by maps . . . . . . . . . . .

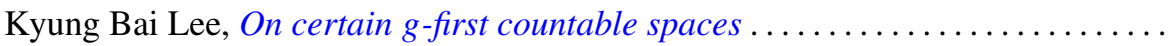

Richard Ira Loebl, A Hahn decomposition for linear maps .................

Moshe Marcus and Victor Julius Mizel, A characterization of functionals on $W_{1}^{p}$ possessing autonomous kernels. I . .

James Miller, Subordinating factor sequences and convex functions of several

variables.

Keith Pierce, Amalgamated sums of abelian l-groups ...

Jonathan Rosenberg, The $C^{*}$-algebras of some real and $p$-adic solvable

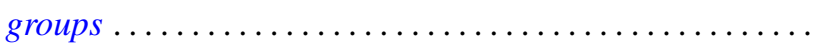

Hugo Rossi and Michele Vergne, Group representations on Hilbert spaces defined

in terms of $\partial_{b}$-cohomology on the Silov boundary of a Siegel domain . .

Mary Elizabeth Schaps, Nonsingular deformations of a determinantal

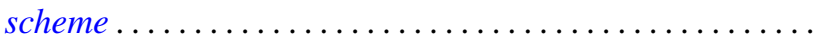

S. R. Singh, Some convergence properties of the Bubnov-Galerkin method...

Peggy Strait, Level crossing probabilities for a multi-parameter Brownian

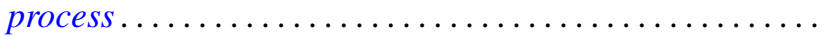

Robert M. Tardiff, Topologies for probabilistic metric spaces .

Benjamin Baxter Wells, Jr., Rearrangements of functions on the ring of integers of

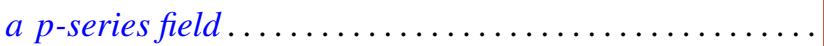

Robert Francis Wheeler, Well-behaved and totally bounded approximate identities for $C_{0}(X)$.

Delores Arletta Williams, Gauss sums and integral quadratic forms over local

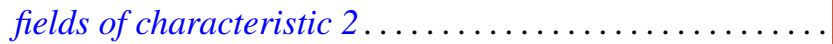

John Yuan, On the construction of one-parameter semigroups in topological 